\title{
Atypical Small Acinar Proliferation and High Grade Prostatic Intraepithelial Neoplasia: Should We Be Concerned? An Observational Cohort Study with a Minimum Follow-Up of 3 Years
}

\author{
Vinaya Srirangam ${ }^{a}$ \\ Bhavan Prasad Raib \\ Ahmed Abroaf ${ }^{b}$ \\ Samita Agarwal ${ }^{a}$ \\ Sergey Tadtayev ${ }^{b}$ \\ Charlotte Foley ${ }^{\mathrm{b}}$ \\ Tim Lane ${ }^{b}$ \\ Jim Adshead ${ }^{\mathrm{b}}$ \\ Nikhil Vasdev ${ }^{b}$

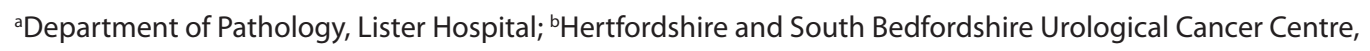 \\ Department of Urology, Lister Hospital, Stevenage, UK
}

\section{Key Words}

Prostatic adenocarcinoma • Prostate biopsy •

Atypical small acinar proliferation .

High grade prostatic intraepithelial neoplasia

\begin{abstract}
Introduction: Atypical small acinar proliferation (ASAP) and high grade prostatic intraepithelial neoplasia (HGPIN) are considered precancerous. We aimed to measure the rate of repeat biopsy and adenocarcinoma in patients with ASAP and HGPIN and identify any clinico-pathologic parameters at diagnosis of ASAP/HGPIN that are predictive of adenocarcinoma. Materials and Methods: Patients with a diagnosis of ASAP/HGPIN with no previous or concomitant cancer were identified. Prostate specific antigen (PSA) and magnetic resonance imaging (MRI) changes were monitored. Re-biopsy was at clinician discretion. Results: Nineteen were diagnosed with ASAP and 17 with HGPIN. Seven with ASAP (37\%) and 6 with HGPIN (35\%) underwent re-biopsy. Three (16\%) with ASAP and 5 with HGPIN (29\%) were diagnosed with adenocarcinoma. The difference in cancer detection rates between ASAP and HGPIN was not significant $(p=0.35)$. Five $(14 \%)$ in total required definitive therapy for adenocarcinoma. Twenty-three (64\%) did not undergo repeat biopsy. Parameters at diagnosis of HGPIN and ASAP, including PSA, prostate vol-
\end{abstract}

\section{KARGER}

Fax +4161306 1234

E-Mail karger@karger.com

www.karger.com
(C) 2016 S. Karger AG, Basel

Accessible online at: www.karger.com/cur ume and PSA density, were compared between the cancer and non-cancer cohorts with none found to be predictive of adenocarcinoma. Conclusion: By monitoring PSA and MRI changes, we managed to spare re-biopsy in two-thirds of patients. Further evaluation is necessary to characterize a surveillance protocol in these populations.

Copyright @ 2016 S. Karger AG, Basel

\section{Introduction}

High grade prostatic intraepithelial neoplasia (HGPIN) and atypical small acinar proliferation (ASAP) are widely considered precancerous lesions in the prostate. Pathologically, prostatic intraepithelial neoplasia involves a continuum of intra-luminal proliferation of atypical secretory cells lining pre-existing ducts and acini in the prostate [1]. ASAP, first defined by Montironi et al. [2], is "a focus of small acinar structures formed by atypical epithelial cells", having some of the features of cancer but not all [3]. Hence, it is a diagnosis of exclusion in cases where suspicious features are present but inadequate to fulfil the diagnostic criteria for cancer [4].

The natural history of both these pathological entities is contentious with conflicting outcomes in contemporary literature. While ASAP is regarded as a high-risk 


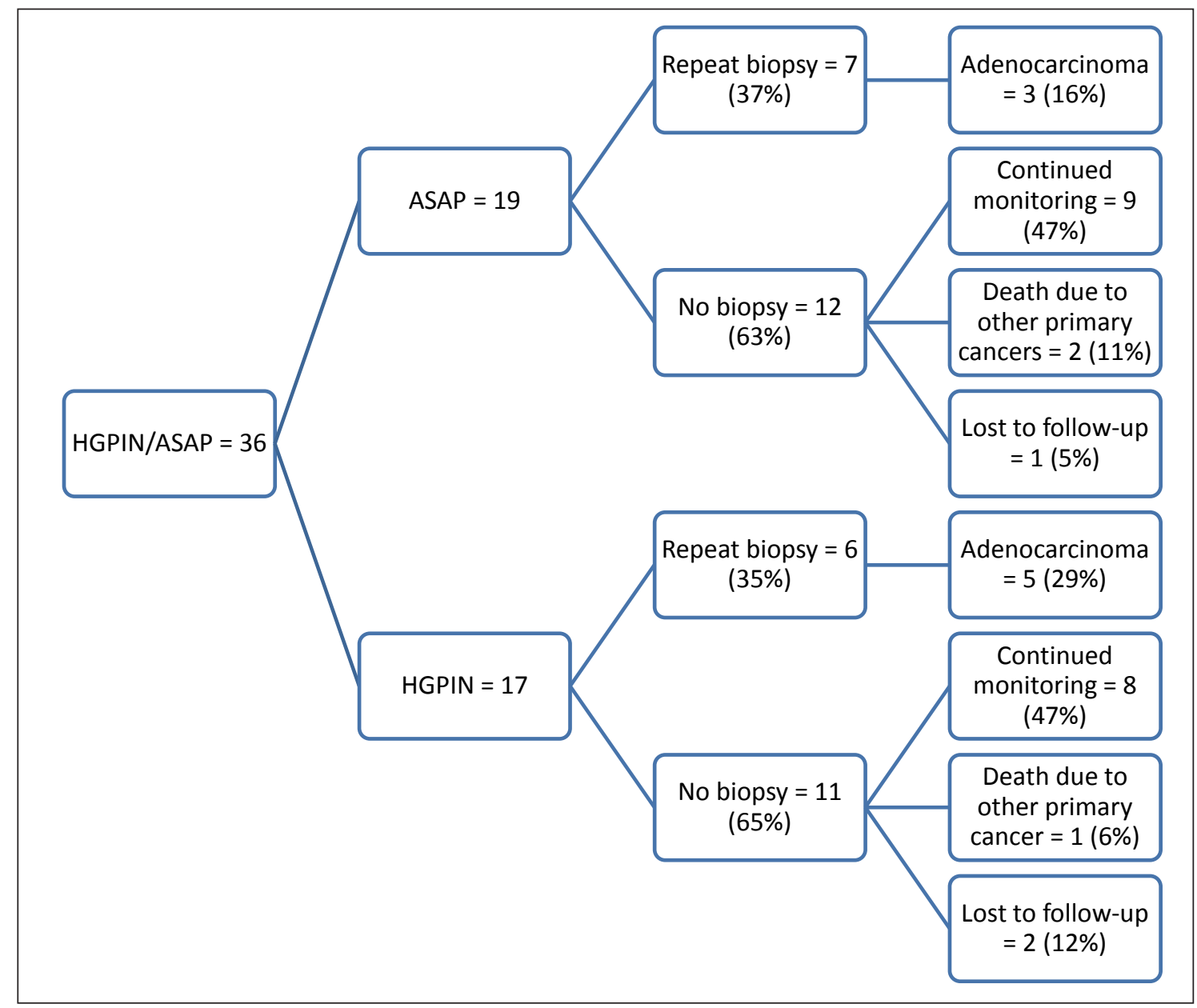

Fig. 1. A diagrammatic representation of the outcomes of patients diagnosed with ASAP and HGPIN.

precursor of cancer, the evidence is less conclusive with respect to HGPIN. Nonetheless, even in the case of the higher-risk ASAP, the clinical significance of cancer detected in this patient group is debatable. Given the morbidity associated with prostate biopsy [5-8], the repeat biopsy protocol instituted for patients with HGPIN and ASAP is of great significance to both clinicians and patients alike. In this study, we aim to evaluate the natural history of patients with ASAP and HGPIN with a midterm follow-up in order to identify the rate of re-biopsy based on prostate specific antigen (PSA) progression and/or magnetic resonance imaging (MRI) changes and identify predictors of prostate adenocarcinoma. We aim to identify a surveillance protocol that can be refined to prevent unnecessary re-biopsy in these patient populations.

\section{Materials and Methods}

A prospective histopathological database with all patients undergoing prostate biopsies has been maintained between 1/1/2011 and 31/12/2012 at the Lister hospital, Stevenage, United Kingdom. Prospectively maintained electronic case notes of all patients were retrospectively reviewed for incomplete data.

All patients underwent transrectal ultrasound-guided prostate biopsy. Indication for prostate biopsy included high age-specific PSA or abnormal digital rectal examination (DRE) findings. Patients whose biopsies showed concomitant prostate cancer or were known to have previous prostate adenocarcinoma were excluded from this study. All patients were followed-up until December 2015. Repeat biopsy was performed at the clinician's discretion based on PSA progression, suspicious changes on MRI with target areas identified for biopsy and patient choice.

Data collected included demographic data, presence of ASAP and HGPIN on initial biopsy, clinico-pathological parameters at diagnosis such as PSA, free or total PSA, abnormalities on DRE 
Table 1. Demographic and clinico-pathological characteristics of patients with ASAP and HGPIN on initial biopsy

\begin{tabular}{|c|c|c|}
\hline & $\begin{array}{l}\text { ASAP } \\
(n=19)\end{array}$ & $\begin{array}{l}\text { HGPIN } \\
(\mathrm{n}=17)\end{array}$ \\
\hline Age, mean (range) & $65.8(44-80)$ & $70.9(58-84)$ \\
\hline PSA at initial biopsy (ng/ml), median (range) & $8(1.1-35)$ & $6(2-15.3)$ \\
\hline $\begin{array}{l}\text { Free/total PSA at initial biopsy (\%), median } \\
\text { (range) }\end{array}$ & $15(5-24)$ & $15(5-18)$ \\
\hline Prostate volume $\left(\mathrm{cm}^{3}\right)$, mean (range) & $46.17(17.3-110)$ & $43.50(20.8-62)$ \\
\hline Abnormal DRE, mean $(\%)$ & $8(42)$ & $5(29)$ \\
\hline $\begin{array}{l}\text { Number of cores at initial biopsy, median } \\
\text { (range) }\end{array}$ & $13(10-31)$ & $16(9-26)$ \\
\hline Number of positive cores, median (range) & $1(1-3)$ & $2(1-26)$ \\
\hline Proportion of positive cores (\%), mean & 10 & 17 \\
\hline $\begin{array}{l}\text { Interval to repeat biopsy (days), median } \\
\text { (range) }\end{array}$ & $364(167-1,183)$ & $524(247-1302)$ \\
\hline $\begin{array}{l}\text { Number of cores at repeat biopsy, median } \\
\text { (range) }\end{array}$ & $23(13-29)$ & $22.5(13-37)$ \\
\hline PSA at repeat biopsy $(\mathrm{ng} / \mathrm{ml})$, median (range) & $7.8(3.9-26)$ & $11.065(7.76-21.1)$ \\
\hline $\begin{array}{l}\text { Difference in PSA between biopsies ( } \mathrm{ng} / \mathrm{ml}) \text {, } \\
\text { median (range) }\end{array}$ & $1.5(2-11)$ & $3.505(1.39-8.97)$ \\
\hline
\end{tabular}

and prostate volume, PSA progression and MRI changes during surveillance and presence of adenocarcinoma on repeat biopsy (Gleason score, number and proportion of cores involved with adenocarcinoma).

\section{Primary Outcomes}

1. Rate of repeat biopsy

2. Rate of adenocarcinoma

3. Fate of patients not undergoing re-biopsy

Secondary Outcomes

We measured the difference between the cancer and noncancer cohorts in the following clinico-pathologic parameters at diagnosis of HGPIN/ASAP:

1. Mean PSA

2. Mean free/total PSA ratio

3. Proportion of patients with abnormal DRE

4. Mean prostate volume

5. Mean PSA density

6. Mean number of positive cores of HGPIN/ASAP

7. Mean proportion of positive cores of HGPIN/ASAP

Statistical Analysis

Mean values were compared with two-sample $t$-tests. Proportion values were compared using the two-tailed $\mathrm{z}$-test $(\mathrm{p}<0.05$ was considered significant).

\section{Results}

Fifty patients were diagnosed with HGPIN and ASAP during the study period. Fourteen patients had previous/ concomitant cancer ( 2 had previous cancers, 12 had cancer on first biopsy) and hence were excluded from the study. Nineteen patients were diagnosed with ASAP and

ASAP/HGPIN

Should We Be Concerned?
17 with HGPIN (fig. 1). None of the patients had both diagnosed concomitantly at initial biopsy. The minimum follow-up period was 3 years. The overall median follow-up was 3.8 years (range $3.0-4.9$ years).

\section{Primary Outcomes: ASAP}

The clinico-pathologic characteristics are summarised in table 1 . The mean age was 65.8 years (range $44-80$ years). The median PSA at initial biopsy was $8 \mathrm{ng} / \mathrm{ml}$ (range $1.1-35 \mathrm{ng} / \mathrm{ml}$ ). The median free/total PSA ratio was 15 (5-24). Eight (42\%) patients had abnormal DRE findings.

Repeat Biopsy Seven (37\%) patients underwent repeat biopsy. The median interval to repeat biopsy was 364 days (range 167-1,183 days). The median PSA at repeat biopsy was $7.8 \mathrm{ng} / \mathrm{ml}$ and the median difference in PSA between re-biopsy and initial biopsy was $1.5 \mathrm{ng} / \mathrm{ml}$. The indications for repeat biopsy were:

- Two $(11 \%)$ patients had PSA progression and target areas identified on MRI for biopsy;

- Two (11\%) patients had only PSA progression with no target areas identified on repeat MRI;

- One (5\%) patient had target areas identified on MRI for biopsy with no PSA progression;

- One $(5 \%)$ patient had persistent low free-to-total PSA (3-5\%) (no MRI performed prior to biopsy);

- One $(5 \%)$ patient had severe anxiety and requested repeat biopsy (no MRI performed prior to biopsy).

Diagnosis of Adenocarcinoma Three (16\%) patients had confirmed prostate adenocarcinoma on repeat biopsy with a Gleason grade of $3+3,3+4$ and $4+4$. These were treated with active surveillance, radical radiotherapy and LHRH therapy respectively (table 2). The patient with cancer of Gleason grade $3+3$ had no suspicious areas identified on MRI, the patient with cancer of grade $3+4$ had target areas identified on MRI and the patient with cancer of grade $4+4$ did not undergo MRI prior to biopsy. Of the remaining 4 patients with repeat biopsy, 2 re-confirmed ASAP (1 underwent MRI with target area suspicious for cancer identified for biopsy, the other did not undergo MRI) and 2 were negative for both ASAP and cancer (both had MRI prior to re-biopsy, with 1 identifying target areas suspicious for cancer and the other identifying no target areas). They continue to be closely monitored.

In total, of the 5 patients who had MRI performed prior to re-biopsy, 3 identified target areas suggestive for cancer with only 1 patient actually diagnosed with cancer on biopsy (of the other two, one was diagnosed with ASAP and the other had a negative result). 
Table 2. Clinico-pathologic characteristics of patients with micro-acinar adenocarcinoma following a diagnosis of ASAP $(n=3)$ or HGPIN $(n=5)$. The below table illustrates the cases of the patients with ASAP first, followed by those with HGPIN. DRE changes are designated as + for positive and - for negative.

\begin{tabular}{|c|c|c|c|c|c|c|c|c|c|c|}
\hline $\begin{array}{l}\text { Age } \\
\text { (year) }\end{array}$ & $\begin{array}{l}\text { PSA at initial } \\
\text { biopsy }(\mathrm{ng} / \mathrm{ml})\end{array}$ & DRE & $\begin{array}{l}\text { Cores with ASAP/ } \\
\text { HGPIN }(\%)\end{array}$ & $\begin{array}{l}\text { Interval to re- } \\
\text { biopsy (days) }\end{array}$ & $\begin{array}{l}\text { PSA on repeat } \\
\text { biopsy }(\mathrm{ng} / \mathrm{ml})\end{array}$ & $\begin{array}{l}\text { Area for target } \\
\text { biopsy on MRI }\end{array}$ & $\begin{array}{l}\text { Cores with } \\
\text { cancer }(\%)\end{array}$ & $\begin{array}{l}\text { Gleason grade/ } \\
\text { laterality }\end{array}$ & $\begin{array}{l}\text { MRI post- } \\
\text { diagnosis }\end{array}$ & Treatment \\
\hline 77 & 15 & - & 6 & 870 & 26 & no MRI & 13 & $\begin{array}{l}4+4=8 \\
\text { bilateral }\end{array}$ & $\begin{array}{l}\text { yes: } \\
\text { organ-confined }\end{array}$ & LHRH \\
\hline 65 & 4.76 & - & 8 & 240 & 4.3 & yes & 38 & $\begin{array}{l}3+4=7 \\
\text { bilateral }\end{array}$ & no & RT \\
\hline 65 & 8.4 & + & 25 & 167 & 16.9 & no target identified & 9 & $\begin{array}{l}3+3=6 \\
\text { unilateral }\end{array}$ & no & AS \\
\hline 74 & 15.3 & + & 6 & 1,036 & 21.13 & yes & 31 & $\begin{array}{l}4+3=7 \\
\text { bilateral }\end{array}$ & $\begin{array}{l}\text { yes: } \\
\text { organ-confined }\end{array}$ & RT, LHRH, AD \\
\hline 67 & 5 & - & 8 & 1,302 & 7.76 & yes & 31 & $\begin{array}{l}3+4=7 \\
\text { unilateral }\end{array}$ & no & RALP \\
\hline 71 & 9.27 & - & 23 & 604 & 10.78 & yes & 4 & $\begin{array}{l}3+3=6 \\
\text { unilateral }\end{array}$ & $\begin{array}{l}\text { yes: no inter- } \\
\text { val change }\end{array}$ & AS \\
\hline 71 & 8.4 & - & 100 & 392 & 9.79 & no MRI & 4 & $\begin{array}{l}3+3=6 \\
\text { unilateral }\end{array}$ & $\begin{array}{l}\text { yes: } \\
\text { organ-confined }\end{array}$ & AS \\
\hline 72 & 5.95 & - & 6 & 247 & 14.92 & yes & 11 & $\begin{array}{l}3+4=7 \\
\text { unilateral }\end{array}$ & $\begin{array}{l}\text { yes: no inter- } \\
\text { val change }\end{array}$ & $\begin{array}{l}\text { perineal prostatec- } \\
\text { tomy, LHRH, AD }\end{array}$ \\
\hline
\end{tabular}

$\mathrm{LHRH}=$ Luteinizing hormone-releasing hormone analogues, $\mathrm{AS}=$ active surveillance, $\mathrm{RT}=$ radiotherapy, $\mathrm{AD}=$ androgen deprivation therapy, $\mathrm{RALP}=$ robot-assisted laparoscopic prostatectomy.

Table 3. Comparison of clinico-pathological parameters at diagnosis of ASAP or HGPIN between the cancer and non-cancer cohorts

\begin{tabular}{|c|c|c|c|c|c|c|}
\hline & \multicolumn{3}{|c|}{ ASAP } & \multicolumn{3}{|c|}{ HGPIN } \\
\hline & Non-cancer $(\mathrm{n}=16)$ & Cancer $(\mathrm{n}=3)$ & $\mathrm{p}$ & Non-cancer $(\mathrm{n}=12)$ & Cancer $(n=5)$ & $\mathrm{p}$ \\
\hline PSA, ng/ml & $10.269 \pm 8.182$ & $9.387 \pm 5.191$ & 0.86 & $6.148 \pm 1.974$ & $8.784 \pm 4.037$ & 0.08 \\
\hline Free/total PSA & $15.1 \pm 5.7$ & $15.5 \pm 3.5$ & 0.91 & $14.2 \pm 5.36$ & $14.6 \pm 6.07$ & 0.89 \\
\hline Proportion of patients with abnormal DRE, \% & 44 & 33 & 0.73 & 33 & 20 & 0.59 \\
\hline Prostate volume, $\mathrm{cm}^{3}$ & $49.33 \pm 24.34$ & $30.36 \pm 14.53$ & 0.21 & $45.03 \pm 15.71$ & $40.08 \pm 6.85$ & 0.51 \\
\hline PSA density, ng/ml.cm ${ }^{3}$ & $0.186 \pm 0.108$ & $0.333 \pm 0.185$ & 0.07 & $0.165 \pm 0.114$ & $0.220 \pm 0.110$ & 0.37 \\
\hline Number of positive cores & $1.4 \pm 0.5$ & $1.6 \pm 1.2$ & 0.62 & $1.8 \pm 0.72$ & $6.4 \pm 11.0$ & 0.15 \\
\hline Proportion of positive cores at diagnosis, $\%$ & 10 & 13 & 0.87 & 12 & 29 & 0.4 \\
\hline
\end{tabular}

ASAP = Atypical small acinar proliferation; HGPIN = high-grade prostatic intraepithelial neoplasia; DRE = digital rectal examination.

Fate of Patients Not Undergoing Re-Biopsy Twelve (63\%) patients did not meet the criteria for repeat biopsy. Nine $(47 \%)$ continued to be monitored, $2(11 \%)$ died from other primary cancers (gastrointestinal and lung) and $1(5 \%)$ was lost to follow-up.

\section{Primary Outcomes: HGPIN}

The clinico-pathologic characteristics are summarised in table 1 . The mean age was 70.9 years (58-84 years). The median PSA at initial biopsy was $6 \mathrm{ng} / \mathrm{ml}(2-15.3$ $\mathrm{ng} / \mathrm{ml})$. The median free/total PSA ratio was 15 (5-18). Five $(29 \%)$ patients had abnormal DRE findings.

Repeat Biopsy Six (35\%) patients underwent repeat biopsy. The median interval to repeat biopsy was 524 days (range 247-1,302 days). The median PSA at repeat biopsy was $11.065 \mathrm{ng} / \mathrm{ml}$ and the median difference in PSA between re-biopsy and initial biopsy was $3.505 \mathrm{ng} /$ $\mathrm{ml}$.

The indication for repeat biopsy was:

- Five $(29 \%)$ patients had PSA progression and suspicious MRI changes with target areas identified for biopsy;

- One (6\%) patients had only PSA progression

Diagnosis of Adenocarcinoma Five (29\%) patients had confirmed prostate adenocarcinoma on repeat biopsy with a Gleason grading of $3+3(n=2), 3+4(n=2)$ and $4+3(n=1)($ table 2$)$. Of these, 4 had target areas identified on MRI and 1 did not undergo MRI prior to biopsy. Of the 5 patients who underwent MRI, 4 subsequently were diagnosed with cancer with one having a negative 
biopsy. The patient with Gleason $4+3$ carcinoma was treated with neoadjuvant androgen deprivation therapy and radical radiotherapy. Both patients with Gleason $3+$ 4 carcinoma underwent radical prostatectomy. The 2 patients with Gleason $3+3$ carcinoma remained on active surveillance, as did the patient with the negative repeat biopsy.

Fate of Patients Not Undergoing Re-biopsy Eleven $(65 \%)$ patients did not undergo re-biopsy. Eight (47\%) continue to be monitored, 2 (12\%) patients were lost to follow-up and $1(6 \%)$ patient died of primary lung cancer.

\section{Secondary Outcomes}

The clinico-pathological parameters at diagnosis of ASAP or HGPIN are presented in table 3. None were significantly different between the cancer and non-cancer cohorts and thus, no parameter was identified as a predictor of adenocarcinoma.

\section{Discussion}

In this study, 36 patients who underwent first time prostate biopsy were diagnosed with either HGPIN or ASAP. The repeat prostate biopsy rate was 36\% (13/36), of which $22 \%(8 / 36)$ of the patients were diagnosed with adenocarcinoma during follow-up with $14 \%(5 / 36)$ requiring radical treatment. A higher proportion of the HGPIN cohort had adenocarcinoma on repeat biopsy compared to the ASAP group (29 vs. $16 \%, \mathrm{p}=0.35$ ). However, this difference was not statistically significant. Twenty-three $(64 \%)$ patients did not require re-biopsy. None of these patients have been adversely affected by prostate adenocarcinoma so far, suggesting that our surveillance protocol could be instituted in wider populations given replicated results.

There has been some speculation in the literature regarding whether factors at diagnosis of ASAP/HGPIN such as PSA [9, 10], prostate volume [11, 12], PSA density $[10,13]$ and number of positive cores [14] can be predictive of adenocarcinoma on repeat biopsy. However, our data is not suggestive of any such relationship between these parameters and subsequent development of adenocarcinoma.

The reported rates of prostate adenocarcinoma on repeat biopsy in contemporary literature are significantly variable, ranging between 30 and 55\% [9-15] for ASAP and 4.5 and $45 \%$ [1] for HGPIN. Differing indications for re-biopsy, biopsy techniques including core sampling techniques and dissimilar study populations between

ASAP/HGPIN

Should We Be Concerned? studies can be ascribed to this observation [16-20]. It is vital that the objective of re-biopsy is to identify carcinoma with clinically significant consequences rather than all carcinomas. The relevance of an initial diagnosis of ASAP or HGPIN with respect to future significant prostate cancer is unclear and the evidence remains conflicting. Dorin et al. [17] reported that a third of patients with ASAP diagnosed with prostate cancer on repeat biopsy within 1 year underwent radical prostatectomy and a quarter underwent radiotherapy, suggesting that early repeat biopsy does identify clinically significant disease. In contrast, Warlick at al. [18] reported only $17.3 \%$ patients to have Gleason 7 or higher disease at a median follow-up of more than 4 years after an initial diagnosis of ASAP. Leone et al. [15] reported that $17 \%$ have intermediate risk cancer and $8 \%$ have high grade cancer on repeat biopsy and recommended active surveillance with monitoring of PSA and DRE changes for patients with ASAP. In our series, only 2 patients $(11 \%)$ with ASAP on initial biopsy had prostate cancer of intermediate or higher grade at repeat biopsy with both undergoing radical therapy.

There is considerable debate as to whether HGPIN is a truly precancerous lesion with potential to progress to cancer. The evidence here is also conflicting with variable advice on the timing of repeat prostate biopsy. Epstein et al. [25] in a review article concluded that patients with HGPIN were at no increased risk of prostate cancer compared to those with benign disease and recommended that repeat biopsy is not required within the first year of the diagnosis of HGPIN. In contrast, Merrimen et al. [1] suggested repeat biopsy at 1 year for patients with multifocal disease and active surveillance thereafter.

The current European Association of Urology guidelines contentiously recommend prostate re-biopsy in all patients with ASAP and extensive HGPIN at initial biopsy [26]. Given the significant morbidity associated with prostate biopsy [5-8], alternate strategies must be considered. There must be clarity on the precise description of extensive HGPIN. Factors such as age, co-morbid status and life expectancy must be taken into consideration before re-biopsy. In this series we have managed to avoid unnecessary repeat prostate biopsies in $64 \%$ of patients.

Notably, there has been a significant uptake of adjuncts such as the multi-parametric MRI in diagnosing significant prostate cancer. Mendhiratta et al. [27] reported in a recent series of 54 patients with an initial diagnosis of HGPIN and ASAP that MRI-ultrasound fusion targeted biopsies identified a significantly larger number of pros- 
tate cancers of Gleason grades 7 or worse, compared to standard systematic prostate biopsies (similar results have been shown by Cool et al. [28]). They concluded that adequate detection of clinically significant cancers with fewer biopsy cores may be achieved in men with a prostate imaging reporting and data system (PI-RADS) score of 4 or higher. Furthermore, they suggested that, in men with previous HGPIN or ASAP, if the MRI scan shows lesions with a PI-RADS score $<4$, a repeat biopsy may be unnecessary. Similarly, a retrospective review by Liddell et al. [29] also suggested that lesions of PIRADS 3 were unlikely to be associated with clinically significant cancer. Raskolnikov et al. [30] reported on MRI-ultrasound fusion targeted re-biopsy in a series of 20 patients at a median of 11.6 months. Five patients were diagnosed with Gleason Grade 3 low-volume prostate cancer. They concluded that, when MRI/trans-rectal ultrasound fusion guided biopsy detected isolated ASAP on index biopsy, early re-biopsy was unlikely to detect clinically significant prostate cancer.

The above studies may provoke questions as to whether HGPIN or ASAP are true precursors to prostate adenocarcinoma. It is possible that both these entities belong to a cluster of abnormal histological abnormalities with no malignant potential at all. The fact that no cancer arose from the ASAP index lesions in the MRI studies would support this hypothesis. Also, prostate biopsies are known to miss a proportion of existing prostate cancer and studies that indeed show an increased incidence of prostate adenocarcinoma on re-biopsy may be identifying cancers that have co-existed with ASAP or HGPIN rather than having developed from them.

Our study is limited by low numbers and hence our results must be viewed with caution. Although one of the strengths of this study is a prospectively maintained his- topathological database and an electronic clinical database, some of the required data was extracted retrospectively, thus introducing a degree of bias. Furthermore, our follow-up did not adopt a standardised protocol and was based on the clinician's discretion. This does introduce a degree of heterogeneity to the follow-up regime. However, this is also a reflection of real life practice in this challenging area. Future research endeavors must concentrate on robust evaluation to establish the longterm natural history of HGPIN and ASAP over a wider population. This is likely to require multicentre, multidisciplinary collaborative research strategies. More work is required in streamlining exactly which patients are at risk of adenocarcinoma, if any at all, and thus keeping re-biopsy rates to a minimum. The role of MRI appears to be promising with respect to the preliminary data and hence requires further evaluation.

\section{Conclusion}

We aimed to monitor PSA and MRI changes in patients with a diagnosis of HGPIN or ASAP with a view to determining the rate of re-biopsy and adenocarcinoma, given the fact that there is some debate regarding the appropriate follow-up and repeat biopsy regimen for this patient population. Given the risks associated with biopsy, both clinicians and patients are keen to reduce the burden of unnecessary biopsy where possible. Only a third of our patients underwent re-biopsy when a surveillance approach was undertaken, as opposed to all with ASAP and widespread HGPIN as recommended by EAU guidelines, suggesting the need for further study to better evaluate a surveillance protocol. 


\section{References}

1 Merrimen JL, Evans AJ, Srigley JR: Preneoplasia in the prostate gland with emphasis on high grade prostatic intraepithelial neoplasia. Pathology 2013;45:251-263.

2 Montironi R, Scattoni V, Mazzucchelli R, Lopez-Beltran A, Bostwick DG, Montorsi F: Atypical foci suspicious but not diagnostic of malignancy in prostate needle biopsies (also referred to as "atypical small acinar proliferation suspicious for but not diagnostic of malignancy"). Eur Urol 2006;50:666-674.

3 Koca O, Caliskan S, Ozturk MI, Gunes M, Karaman MI: Significance of atypical small acinar proliferation and high-grade prostatic intraepithelial neoplasia in prostate biopsy. Korean J Urol 2011;52:736-740.

4 Iczkowski KA: Current prostate biopsy interpretation: criteria for cancer, atypical small acinar proliferation, high-grade prostatic intraepithelial neoplasia, and use of immunostains. Arch Pathol Lab Med 2006;130:835843.

5 Loeb S, Vellekoop A, Ahmed HU, Catto J, Emberton M, Nam R, Rosario DJ, Scattoni V, Lotan Y: Systematic review of complications of prostate biopsy. Eur Urol 2013;64:876892.

6 Loeb S, Carter HB, Berndt SI, Ricker W, Schaeffer EM: Is repeat prostate biopsy associated with a greater risk of hospitalization? Data from SEER-Medicare. J Urol 2013;189: 867-870.

7 Lundström KJ, Drevin L, Carlsson S, Garmo H, Loeb S, Stattin P, Bill-Axelson A: Nationwide population based study of infections after transrectal ultrasound guided prostate biopsy. J Urol 2014;192:1116-1122.

8 Quaresima L, Lacetera V, Leone L, Montesi L, Cantoro U, Polito M, Muzzonigro G: The impact of repeated prostate biopsies on sexual function and urinary symptoms in patients with diagnosis of atypical small acinar proliferation (ASAP): can ecoDoppler reduce side effects? Arch Ital Urol Androl 2014;86: 356-358.

9 Moore CK, Karikehalli S, Nazeer T, Fisher HA, Kaufman RP, Mian BM: Prognostic significance of high grade prostatic intraepithelial neoplasia and atypical small acinar proliferation in the contemporary era. J Urol 2005;173:70-72.

10 Abdel-Khalek M, El-Baz M, Ibrahiem el-H: Predictors of prostate cancer on extended biopsy in patients with high-grade prostatic intraepithelial neoplasia: a multivariate analysis model. BJU Int 2004;94:528-533.

11 Mearini L, Costantini E, Bellezza G, Cavaliere A, Zucchi A, Bini V, Porena M: Is there any clinical parameter able to predict prostate cancer after initial diagnosis of atypical small acinar proliferation? Urol Int 2008;81:29-35.
12 Scattoni V, Roscigno M, Freschi M, Dehò F, Raber M, Briganti A, Fantini G, Nava L, Montorsi F, Rigatti P: Atypical small acinar proliferation (ASAP) on extended prostatic biopsies: predictive factors of cancer detection on repeat biopsies. Arch Ital Urol Androl 2005; 77:31-36

13 Amin MM, Jeyaganth S, Fahmy N, Bégin L, Aronson S, Jacobson S, Tanguay S, Aprikian AG: Subsequent prostate cancer detection in patients with prostatic intraepithelial neoplasia or atypical small acinar proliferation. Can Urol Assoc J 2007;1:245-249.

14 Kim TS, Ko KJ, Shin SJ, Ryoo HS, Song W, Sung HH, Han DH, Jeong BC, Seo SI, Jeon SS, Lee KS, Lee SW, Lee HM, Choi HY, Jeon HG: Multiple cores of high grade prostatic intraepithelial neoplasia and any core of atypia on first biopsy are significant predictor for cancer detection at a repeat biopsy. Korean J Urol 2015;56:796-802.

15 Leone A, Gershman B, Rotker K, Butler C, Fantasia J, Miller A, Afiadata A, Amin A, Zhou A, Jiang Z, Sebo T, Mega A, Schiff S, Pareek G, Golijanin D, Yates J, Karnes RJ, Renzulli J: Atypical small acinar proliferation (ASAP): Is a repeat biopsy necessary ASAP? A multi-institutional review. Prostate Cancer Prostatic Dis 2016;19:68-71.

16 Leone A, Rotker K, Butler C, Mega A, Li J, Amin A, Schiff SF, Pareek G, Golijanin D, Renzulli JF 2nd: Atypical small acinar proliferation: repeat biopsy and detection of high grade prostate cancer. Prostate Cancer 2015; 2015:810159.

17 Dorin RP, Wiener S, Harris CD, Wagner JR: Prostate atypia: does repeat biopsy detect clinically significant prostate cancer? Prostate 2015;75:673-678.

18 Warlick C, Feia K, Tomasini J, Iwamoto C, Lindgren B, Risk M: Rate of Gleason 7 or higher prostate cancer on repeat biopsy after a diagnosis of atypical small acinar proliferation. Prostate Cancer Prostatic Dis 2015;18: 255-259.

19 Leone L, Lacetera V, Montironi R, Cantoro U, Conti A, Sbrollini G, Quaresima L, Mariani L, Muzzonigro G, Galosi AB: Biopsy follow-up in patients with isolated atypical small acinar proliferation (ASAP) in prostate biopsy. Arch Ital Urol Androl 2014;86:332335.

20 Laurila M, van der Kwast T, Bubendorf L, di Lollo S, Pihl CG, Ciatto S, Hugosson J, Määttänen L, Roobol MJ, Kujala PM: Detection rates of cancer, high grade PIN and atypical lesions suspicious for cancer in the European Randomized Study of Screening for Prostate Cancer. Eur J Cancer 2010;46:30683072.
21 Tan PH, Tan HW, Tan Y, Lim CN, Cheng C, Epstein JI: Is high-grade prostatic intraepithelial neoplasia on needle biopsy different in an Asian population: a clinicopathologic study performed in Singapore. Urology 2006; 68:800-803.

22 Singh PB, Nicholson CM, Ragavan N, Blades RA, Martin FL, Matanhelia SS: Risk of prostate cancer after detection of isolated high-grade prostatic intraepithelial neoplasia (HGPIN) on extended core needle biopsy: a UK hospital experience. BMC Urol 2009;9:3.

23 Soos G, Tsakiris I, Szanto J, Turzo C, Haas PG, Dezso B: The prevalence of prostate carcinoma and its precursor in Hungary: an autopsy study. Eur Urol 2005;48:739-744.

24 Sánchez-Chapado M, Olmedilla G, Cabeza M, Donat E, Ruiz A: Prevalence of prostate cancer and prostatic intraepithelial neoplasia in Caucasian Mediterranean males: an autopsy study. Prostate 2003;54:238-247.

25 Epstein JI, Herawi M: Prostate needle biopsies containing prostatic intraepithelial neoplasia or atypical foci suspicious for carcinoma: implications for patient care. J Urol 2006; 175:820-834.

26 Heidenreich A, Bastian PJ, Bellmunt J, Bolla $\mathrm{M}$, Joniau $\mathrm{S}$, van der Kwast $\mathrm{T}$, Mason $\mathrm{M}$, Matveev V, Wiegel T, Zattoni F, Mottet N: EAU guidelines on prostate cancer. part 1: screening, diagnosis, and local treatment with curative intent-update 2013. Eur Urol 2014;65:124-137.

27 Mendhiratta N, Rosenkrantz AB, Meng X, Fenstermaker M, Huang R, Wysock JS, Deng FM, Zhou M, Huang WC, Lepor H, Taneja SS: MP77-18 outcomes of MRI-US fusion targeted prostate biopsy in men with history of prostatic intraepithelial neoplasia and/or atypical small acinar proliferation: evidence for an alteration of the current practice. J Urol 2015;193:e1000-e1000.

28 Cool DW, Romagnoli C, Izawa JI, Chin J, Gardi L, Tessier D, Mercado A, Mandel J, Ward AD, Fenster A: Comparison of prostate MRI-3D transrectal ultrasound fusion biopsy for first-time and repeat biopsy patients with previous atypical small acinar proliferation. Can Urol Assoc J 2016;10:342-348.

29 Liddell H, Jyoti R, Haxhimolla HZ: mp-MRI prostate characterised PIRADS 3 lesions are associated with a low risk of clinically significant prostate cancer - a retrospective review of 92 biopsied PIRADS 3 lesions. Curr Urol 2015;8:96-100.

30 Raskolnikov D, Rais-Bahrami S, George AK, Turkbey B, Shakir NA, Okoro C, Rothwax JT, Walton-Diaz A, Siddiqui MM, Su D, Stamatakis L, Yan P, Kruecker J, Xu S, Merino MJ, Choyke PL, Wood BJ, Pinto PA: The role of image guided biopsy targeting in patients with atypical small acinar proliferation. J Urol 2015;193:473-478. 